\title{
Infectious Diseases in Children, Never a Dull Moment!
}

\author{
Aracelis D. Fernandez ${ }^{1} \cdot$ Narendra Rathi $^{2}$
}

Received: 19 June 2020 / Accepted: 19 June 2020 / Published online: 13 July 2020

(C) Dr. K C Chaudhuri Foundation 2020

In the midst of COVID-19 for some countries and in the aftermath for others, we are starting to see more children of all ages being affected by COVID-19 and some severe cases of the newly described Multisystem Inflammatory Syndrome in Children (MIS-C) [1]. Globalization does not know about borders and nowadays infections travel very fast. Even though over the last several months COVID-19 has deservedly dominated the news worldwide by overwhelming healthcare systems with almost 8 million confirmed cases and almost 450,000 deaths worldwide in mid-June [2], and crippling the world economy, other infections deserve attention and need to be not forgotten particularly now that resources are severely limited and they could fuel other situations of great public health concerns.

A series of review articles are featured in the Symposium on Reemerging Infections including Influenza, MDR and XDR Tuberculosis, Congenital Cytomegalovirus and Zika Infections, Rickettsioses in Children, Antibiotic Resistance- A Cause for Reemergence of Infections, and "Rationalization of Empiric Antibiotic Therapy" - A Move Towards Preventing Emergence of Resistant Infections and are a reminder of a few infections which can have devastating consequences.

As Dr. Dharmapalan reminds us in his excellent influenza review, over 100 years ago the world encountered the worst pandemic ever, as the 1918 Spanish flu killed more than 50 million people. Influenza continues to be a significant source of morbidity and mortality throughout the years despite the tools we have available to prevent and treat it. Luckily, new

Aracelis D. Fernandez

aracelis.fernandez@nychhc.org

1 Department of Pediatrics, Pediatric Infectious Diseases, NYC Health + Hospitals/Harlem, Affiliate of Columbia University College of Physicians and Surgeons, 506 Lenox Ave, MLK 16-138, New York, NY 10037, USA

2 Smile Institute of Child Health, Ramdaspeth, Akola, Maharashtra 444001, India drugs including the recently FDA approved Baloxavir marboxil and the 2 intravenous peramivir and azamivir in clinical trials will afford us more treatment options. Nevertheless the best treatment is still prevention with vaccine use, good hand hygiene and cough etiquette [3]. These are the best ways of preventing/controlling influenza activity. One sure thing COVID-19 has highlighted is the power of hand hygiene and cough etiquette among other social and personal effective measures to prevent certain types of infections.

In another excellent article of this symposium, Dr. Shah and co-authors reviewed MDR and XDR tuberculosis. In 2018, the world saw 1.5 million people died from tuberculosis as it continues to be the leading cause of death from a single infectious agent [4]. MDR and XDR tuberculosis are a growing concern as their prevalence has increased particularly in high burden countries making the treatment and control of the disease more difficult and costly [5]. We can't underestimate how important it is to early identify, isolate, trace contacts and treat patients with effective and safe anti-tuberculosis medications. Rapid diagnostic tests detecting resistance such as the cartridge based nucleic acid amplification test, line probe assay, TrueNat, next generation sequencing including whole genome sequencing are a valuable aid to the conventional microscopy and drug susceptibility testing by culture based methods. Newer drugs for the treatment of drug-resistant tuberculosis in children include linezolid, bedaquiline, and delamanid. Other drugs to treat tuberculosis being studied are delpazolid, contezolid, macozinone, pretomanid, sutezolid, OPC-167832, Q203, TBA-7371, TBI-166, GSK3036656, and SQ109 [5]. These new drugs provide some sense of relief as we face the difficult challenges of treating MDR and XDR tuberculosis.

Dr. Angueyra and co-authors contributed an excellent review of 2 viruses that can negatively affect neonates including their developmental and intellectual abilities: Cytomegalovirus (CMV) and Zika infections. Congenital CMV is the most common congenital infection and even though most neonates are asymptomatic at birth, this virus is the most common cause of non-hereditary sensorineural hearing loss; while congenital Zika 
infection causes severe microcephaly and neurodevelopmental delays in affected infants. Unfortunately, currently available antiviral medications are not effective against Zika virus; luckily for infants with moderate to severe symptoms of congenital CMV infection, prolonged treatment with valganciclovir has shown benefits albeit with associated potential side effects [6]. While clinical trials are being conducted to assess the safety and efficacy of vaccines, antivirals and immunoglobulins, at this time the best approach to manage these 2 congenital infections is to prevent their acquisition and to establish an early diagnosis and close developmental monitoring and follow up.

Due to the high morbidity, difficulty in confirming the diagnosis, adverse outcomes including mortality if not properly and promptly treated, Rickettsial diseases have emerged as a serious public health problem in certain parts of the world [7]. Dr. Sood provides us with an excellent review of this illness and its different clinical manifestations, diagnosis challenges, and treatment as well as 2 helpful tables for quick reference [7].

A symposium on infectious diseases is not completed without addressing the major problem that antibiotic resistance poses to health care systems in general and health of individuals in particular. Two excellent articles to address this problem are included in the symposium. Antimicrobial resistance is a significant cause of disease and death burden worldwide and it is considered a global threat with good reasons as more than 58,000 babies died in India in one year secondary to infections with resistant bacteria acquired from their mothers and this is only one country example. Different factors contribute to the emergence of antimicrobial resistance, including explosion in human population leading to crowded urban centers coupled with the ability to be anywhere in the world in a limited amount of time providing the venue for illnesses and resistant microbes to come along. Poor hygiene and sanitation practices in rural and urban settings alike, substandard infection control practices in hospitals, clinics, nursing homes, lack of rapid laboratory tests available widely to promptly identify pathogens and best antimicrobial agents, and antimicrobial use in agriculture also lead to antimicrobial resistance. Patients and medical providers both play a major role with providers over-prescribing antibiotics and patients not taking antibiotics as prescribed [8] or in many instances, self-prescribing antibiotics in certain parts of the globe where antibiotics can be purchased in local grocery stores. Dr. Subramaniam and Dr. Girish share guidelines on antimicrobial use in their contribution to the symposium. They briefly address the use of antibiotics in upper respiratory infections, their use in diarrhea, guiding empiric antibiotic treatment, shorter courses of antibiotics, and the role of vaccines in the prevention of antimicrobial resistance. Equally important in dealing with this threat is the implementation of antimicrobial stewardship programs both in inpatient and outpatient settings, and the development and access to rapid diagnostic guiding the antibiotic therapy [9]. Dr. Singhal's article nicely complements this part of the symposium by providing a detailed empiric antimicrobial therapy guidance from asking the first question "Is Empiric Therapy Required?" to discussing the principles guiding the empiric therapy and different common clinical scenarios [10].

\section{Compliance with Ethical Standards}

Conflict of Interest None.

\section{References}

1. World Health Organization MIS-C Case Definition (2020). Available at: https://www.who.int/news-room/commentaries/ detail/multisystem-inflammatory-syndrome-in-children-andadolescents-with-covid-19. Accessed 10 June 2020.

2. World Health Organization COVID Dashboard. Available at: https://Covid19.who.int/. Accessed 10 June 2020.

3. Dharmapalan D. Influenza. Indian J Pediatr. 2020. https://doi.org/ 10.1007/s12098-020-03214-1.

4. World Health Organization Tuberculosis. 2020. Available at: https:/www.who.int/news-room/fact-sheets/detail/tuberculosis. Accessed 11 June 2020.

5. Shah I, Poojari V, Meshram H. Multi-drug resistant and extensively-drug resistant tuberculosis. Indian J Pediatr. 2020. https://doi.org/10.1007/s12098-020-03230-1.

6. Angueyra C, Hatab HA, Pathak A. Congenital cytomegalovirus and zika infections. Indian J Pediatr. 2020. https://doi.org/10.1007/ s12098-020-03260-9.

7. Sood AK, Sachdeva A. Rickettsioses in children - a review. Indian J Pediatr. 2020. https://doi.org/10.1007/s12098-020-03216-z.

8. Center for Diseases Control and Prevention Antibiotic Resistance Infographic 2020. Available at: https:/www.cdc.gov/globalhealth/ infographics/antibiotic-resistance/antibiotic_resistance_global_ threat.htm Accessed 12 June 2020.

9. Subramaniam G, Girish M. Antibiotic resistance - a cause for reemergence of infections. Indian J Pediatr. 2020. https://doi.org/10. 1007/s12098-019-03180-3.

10. Singhal T. "Rationalization of empiric antibiotic therapy" - a move towards preventing emergence of resistant infections. Indian J Pediatr. 2020. https://doi.org/10.1007/s12098-019-03144-7.

Publisher's Note Springer Nature remains neutral with regard to jurisdictional claims in published maps and institutional affiliations. 